\title{
Design of a computational model for multi-body contact of deformable bodies in the flowing fluid
}

\author{
Marián Handrik ${ }^{1, *}$, Milan Vaško ${ }^{1}$ \\ ${ }^{1}$ University of Žilina, Faculty of Mechanical Engineering, Department of Applied Mechanics, \\ Univerzitná 8215/1, 01026 Žilina, Slovak Republic
}

\begin{abstract}
The article deals with the design and testing of a computational model for multi-body contact of deformable bodies in the flowing fluid. The computational model will be designed to allow easy modify the impact area of deformable bodies and their shape. The computational model must allow the application of an endless cycle of bodies impact with the possibility of restarting and calculating the following time intervals for collision of bodies.
\end{abstract}

Keywords: multi-body contact, fluid structure interaction, finite element method, ADINA

\section{Modelling of multi-body contact in the fluid flow}

Modelling of a multi-body contact is a computationally demanding task. The computational difficulty of a model increases with a number of bodies that may come into contact. If we consider a possible contact among all bodies in the solved task, then a number of contact pairs will be determined according to the relation $\mathrm{P}=(\mathrm{n}-1)^{2}$, where $\mathrm{n}$ is the number of all bodies in the contact problem. When generating a mesh of finite elements, it is necessary to define a sufficiently dense mesh of finite elements on the contact bodies. The contact task has bad convergence in case of low mesh density. Mesh density must be also determined with regard to shape complexity of bodies in such a way it approximates an edge of a contact body with sufficient precision. Most of computational models of the contact implemented in software for computing by the method of finite elements divide contact bodies into a group of 'contactor" and 'target". In such a case the contact is detected in case when nodal points of the body 'contactor' penetrate into a body of the type 'target'. On the contrary, if nodal points of the mesh of a body 'target' penetrate into a body of 'contactor', a contact is not detected. In case of a multi-body contact it is convenient to define contact pairs in such a way that each body is a 'contactor' and at the same time also a 'target'. By such a definition of contact pairs convergence of a solution of the contact task becomes rapidly improved and oscillation in nodal points is decreased [1-3].

\footnotetext{
* Corresponding author: marian.handrik@,fstroj.uniza.sk

Reviewers: Bohuš Leitner, Tomáš Lack
} 
Computational models for solving of flowing fluids require a fine mesh of elements, elements must be sufficiently small so that a flow in the boundary layer close to a body surface would be possible to solve. Both groups of computational models, contact tasks and flow, belong to a group of models with a high demand on computational means and time [4-9].

The aim of the design of a computational model is to design an efficient computational model for the analysis of a mutual contact of bodies in flowing fluid [10-11]. The designed computational model must contain following properties:

- A part of the model must be constructed in a way to enable mutual collisions of contact bodies and a shape of the area must be easily modified.

- A channel in which fluid flows and carries contact bodies must be closed, bodies proceed through the channel and re-collide.

- A computational model must be maximally efficient from the standpoint of requirements on computational means and computing time.

- A designed computational model will be created for the software package ADINA, a designed model will consider possibilities of a model design in this program.

- Contact bodies will have a circular shape.

- A shape of the channel must be designed to avoid mutual jamming and a blockage of the channel.

- A task will be solved in 2D while structural bodies will have plane state of stress.

- In flowing, a material model for viscous flow regardless a turbulence model will be considered.

In the system ADINA it is necessary to design an individual geometric model and finite element model for a structural analysis and an individual model for the flow analysis. A calculation is performed as an iterative process to search for a balance between the structural analysis and the flow analysis in every time step of the solution. A mutual interaction between models is transferred from the structural model into the model of the flow by means of a change of a position of limits if bodies in the fluid. Active forces determined from pressure on limits of bodies are transferred from the model of the flow into the structural model.

\section{The geometric and FE model for the structural analysis}

The designed geometric model of an enclosed channel in the structural analysis is formed by the following elements. The geometric model is shown in Figure 1:

- A structure forming an outer boundary of the channel. Geometry of the body is designed in the system ADINA via an application of the module ADINA-M which is the module for modelling of bodies by the use of a library for a graphic format parasolid. A gradual shape of the channel is designed in a way to prevent mutual jamming of contact bodies and the channel blockage.

- The structure forming an inner boundary of the channel. As in the case of the outer boundary, even the inner boundary is designed by the application of the module ADINA-M.

- Bodies in the flowing fluid. Circular bodies are divided into four segments, i.e., quarters of the circle. Bodies are designed by this method, so that it would be possible to minimize a number of elements generated on the bodies by an application of a mapping mesh by triangle elements.

- An obstacle in the centre of a widened part of the channel. Bodies stop by the obstacle and thus a mutual contact is formed. The body is formed in the module ADINA-M.

- A system of auxiliary lines enables a definition of a movement of contact bodies in the channel. The system consists of a set of lines that are meshed by the use of beam 
elements. A rotation of auxiliary points moves contact bodies in the channel. A speed of rotation is defined thus the speed of movement of contact bodies is $0.1 \mathrm{~ms}^{-1}$ [12-13].

A diameter of contact bodies is $10 \mathrm{~mm}$, the mean diameter of the circle in curves of the channel is $60 \mathrm{~mm}$ and the width of the channel is $11 \mathrm{~mm}$.

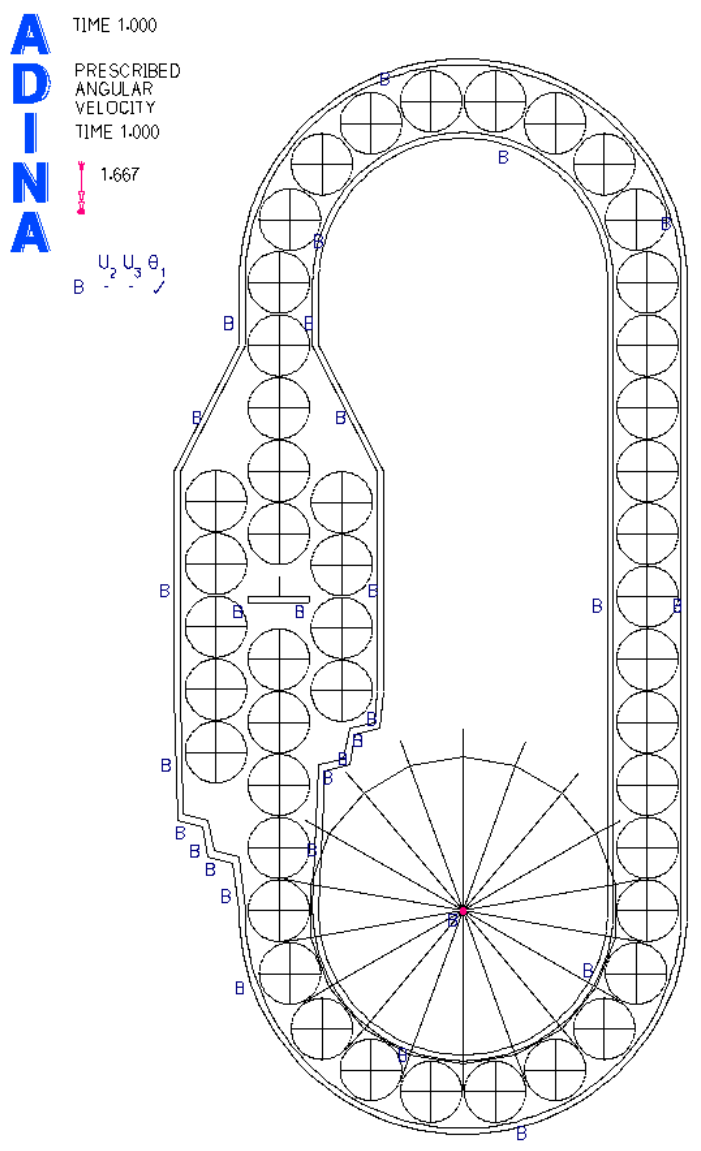

Fig. 1. Geometry of structural model and boundary condition

The program ADINA enables a definition of several contact groups, in a contact group contact bodies and contact pairs are defined. Due to less complex modelling, a design of a model, we defined following contact groups:

- The first contact group - a mutual contact of contact bodies. A geometric model contains 46 circular contact bodies. In case of a potential contact of each body with all bodies there are designed 2116 contact pairs in the model.

- The second contact group - a contact of 46 circular contact bodies and obstacles in a channel. The contact group contains 92 contact pairs. Each body is defined as a 'contactor' and a 'target' at the same time.

- The third contact group - a contact of 46 circular contact bodies and an outer boundary of the channel. There are 92 contact pairs in this contact group.

- The fourth contact group - a contact of 46 circular contact bodies with an inner boundary of the channel. There are 92 contact pairs in this group. 
- The fifth contact group - a contact of 46 circular contact bodies and 18 contact bodies of auxiliary geometry. There are 828 contact pairs in this group.

In the first four contact groups we must define an occupation of contact areas within small distances and we use the value $5 \times 10^{-2} \mathrm{~mm}$ in the model while the diameter of contact bodies is $10 \mathrm{~mm}$. Occupation of a body contact is necessary for a correct function of the model of flow. Among contact bodies there must be formed a thin layer of flowing elements - at least one element. Otherwise, it would not be possible to generate a mesh of finite elements for a model of the flowing fluid, boundaries of an area in the model of flowing must not overlap or contact.
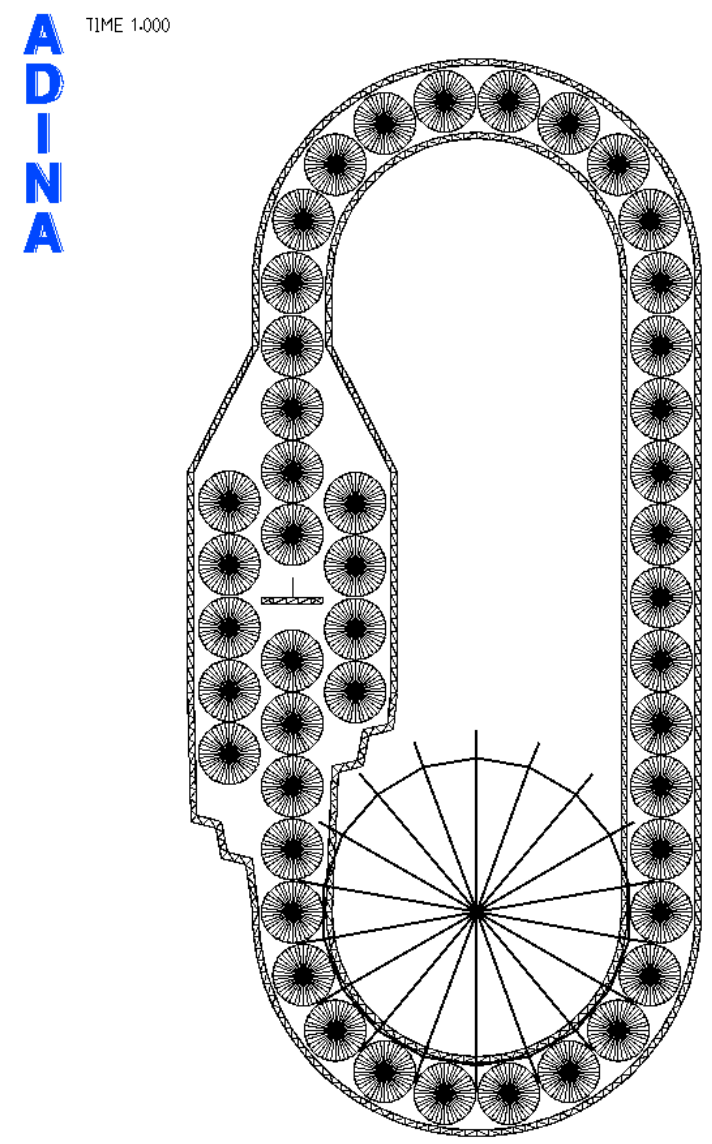

Fig. 2. Finite element mesh of structural model

In the designed structural model there is applied an elastic material model for steel, Young's modulus of elasticity $2.1 \times 10^{11} \mathrm{~Pa}$, Poisson number 0.3 , density $7850 \mathrm{~kg} \mathrm{~m}^{-3}$.

In the model following initial and boundary conditions are defined:

- A withdrawal of all stages of independence on an outer side of the boundary of the channel, they fix a position of the channel in the space.

- In the centre of auxiliary geometry, shifts in a direction of axes $\mathrm{y}$ and $\mathrm{z}$ are removed, a rotation in a direction of the axis $\mathrm{x}$ remains.

- A removal of shifts in two points on the obstacle in the channel, it fixes a position of the obstacle in the space. 
- A load by gravitation in a direction of $z$ (in negative direction).

- A definition of an angular velocity in a centre of auxiliary geometry1.6666 $\mathrm{rads}^{-1}$. Contact bodies will have the speed of $0.1 \mathrm{~ms}^{-1}$.

- A zero initial speed is defined for all bodies.

A calculation is solved as an implicit task by Newmark method, for plane stress state a thickness of designed geometric model is $100 \mathrm{~mm}$. Thickness of $100 \mathrm{~mm}$ is selected thus maximum contact forces will not exceed $100 \mathrm{~N}$. Properties of the contact are selected in a way that contact forces lower than $0.1 \mathrm{~N}$ are not considered in the contact. Limits of a minimum value of contact forces strikingly improve convergence of a contact task and it prevents oscillation in contact detection.

Due to a reason of the maximum efficiency of a computing model we selected a mesh density to generate a minimum number of elements in which a convergence of the task is secured. In this case values of contact forces are calculated correctly, but the state of stress in the contact point is not. In a case we would intend to determine the state of stress with sufficient precision in the contact point, i tis necessary to increase density and, eventually, to change a type of elements into quadratic elements. In generating of a mesh on contact bodies we use 10 elements per a quarter of a circle. In the other parts of the model, the size of elements is set on $2 \mathrm{~mm}$. Auxiliary lines are modelled via an application of one beam element per a line. The final part of the auxiliary lines is modelled with a small cross section of the beam and beam elements form an elastic element in this case. A presence of an elastic element in the model decreases maximum values of contact forces among circular contact bodies and auxiliary lines in an application of greater time span [14]. A mesh of linear triangular elements for a structural model is depicted in Figure 2.

\section{The geometric and FE model for analysis of fluid flow}

When designing a geometric model of flow we follow geometry designed for the structural model. For designing an outer boundary of the area boundary curves from the structural model are applied and they are supplemented by the inflow and outflow parts of the model. Boundaries of contact bodies are defined by a construction of a circle with a rotating point of $360^{\circ}$. Consequently, bodies in the module of ADINA-M are designed from these boundary curves and the geometric model for a boundary in the area of fluid in the model is formed by their mutual subtracting. The geometric model is depicted in Figure 3.

In the model there is applied a model of a laminar flow and following properties of the fluid (water) are defined: dynamic viscosity $0.001 \mathrm{kgm}^{-1} \mathrm{~s}^{-1}$, fluid density $1000 \mathrm{~kg} \mathrm{~m}^{-3}$.

In the designed model of flow there are defined following boundary and initial conditions:

- The boundary of contact bodies is defined as ,,no slip FSI boundary“.

- In all the parts of the outer boundary of the area of flowing is defined as no slip boundary condition.

- In the inflow and outflow areas there is defined the velocity of fluid flow $0.1 \mathrm{~ms}^{-1}$.

- In the model there is defined the initial condition with zero speed of flow in all the points of the mesh of finite elements.

To solve the issue Euler's method for solving transactional analysis of fluid flow is applied. When designing a mesh of finite elements the FCBI-C formulation of elements for flow is applied. In FCBI-C formulation of finite elements is defined one control point in the centre of gravity in every element. FCBI-C formulation enables an application of a segregated iteration solver AMG. A selection of a formulation of elements and a type of a solver is designated with a regard of minimisation of a time period needed for a solution. In meshing 
we used triangle elements of the size $0.2 \mathrm{~mm}$ and in the process of solution the automatic meshing of finite elements for flow is activated.

In solving, the constant time step $1 \times 10^{-4} \mathrm{~s}$ is applied.

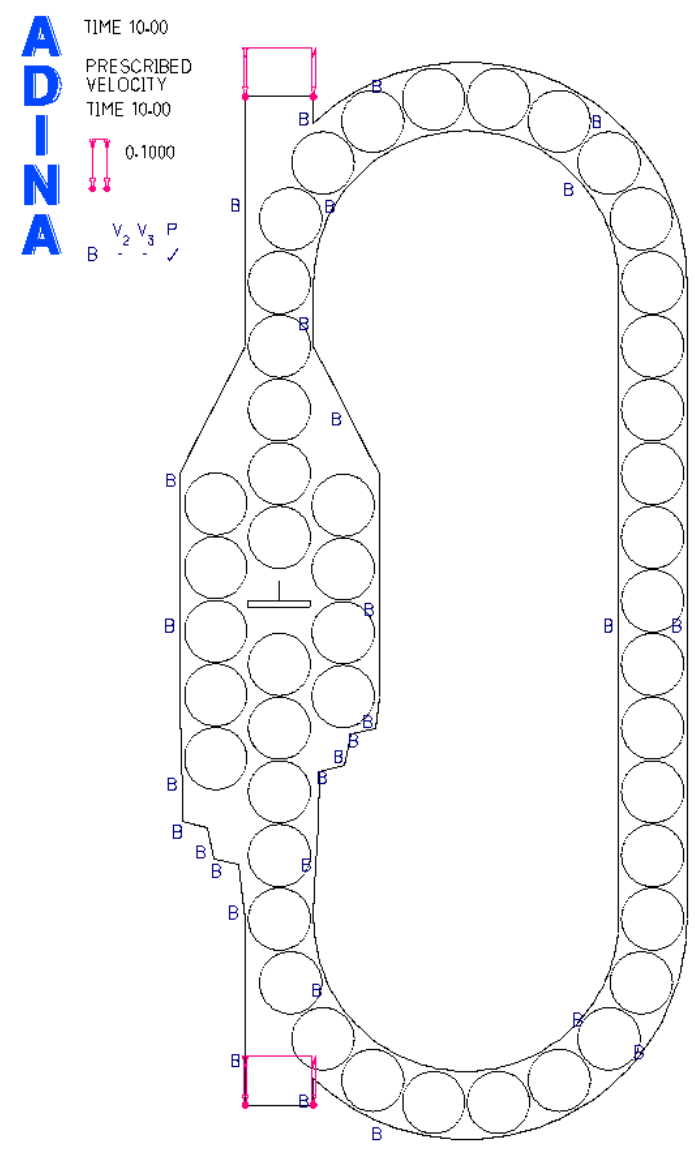

Fig. 3. Geometry of fluid model and boundary condition

\section{The solution for the model of multi-body contact of bodies in the flowing fluid}

When solving FSI analysis via FCBI-C elements the iteration scheme for finding the balance of the structural analysis and analysis of flow is modified in the following way. The calculation is started by a determination of a balance in the structural analysis, then there follows one outer iteration of AMG solver for computation of the model of flow, a procedure repeats and thus the preciseness of a solution of the model of flow improves. The iteration process of finding of a balance in FSI analysis is completed when the prescribed preciseness of the solution of the model of flow or the maximum number of iteration of AMG solver for flow are reached. 
The designed computing model has following properties from the standpoint of a solution of a task of multi-body contact of bodies in the flowing fluid:

- The number of iterations of the Newton-Raphson method for a solution of a contact task in one time step was within the interval 3 to 25 iterations, exceptionally and more iterations were necessary.

- The number of iteration for finding the balance of a solution of interaction of a construction of a body with fluid ranged within the interval 2 to 30 outer iterations of AMG solver, in rare cases the model of flow did not converge even by 250 iterations. In the next time step the standard speed of task convergence was reached within the interval $2-30$ iterations.

- The task was solved on the server IBM dx360 M 3. 2 x CPU Intel I7 $2.4 \mathrm{GHz} 6$ cores, 96 GB RAM. In computing, parallelisation of the program ADINA of the type SMP and 12 computing nucleus were applied. Maximally, 2.5 GB RAM was used. The period of solution of one time increment of the solved task ranged within the interval $3-40$ seconds according to a number of iterations. 180 seconds were necessary for a total meshing of the model of flow. Meshing of the model was necessary after approx. 80 time increments.

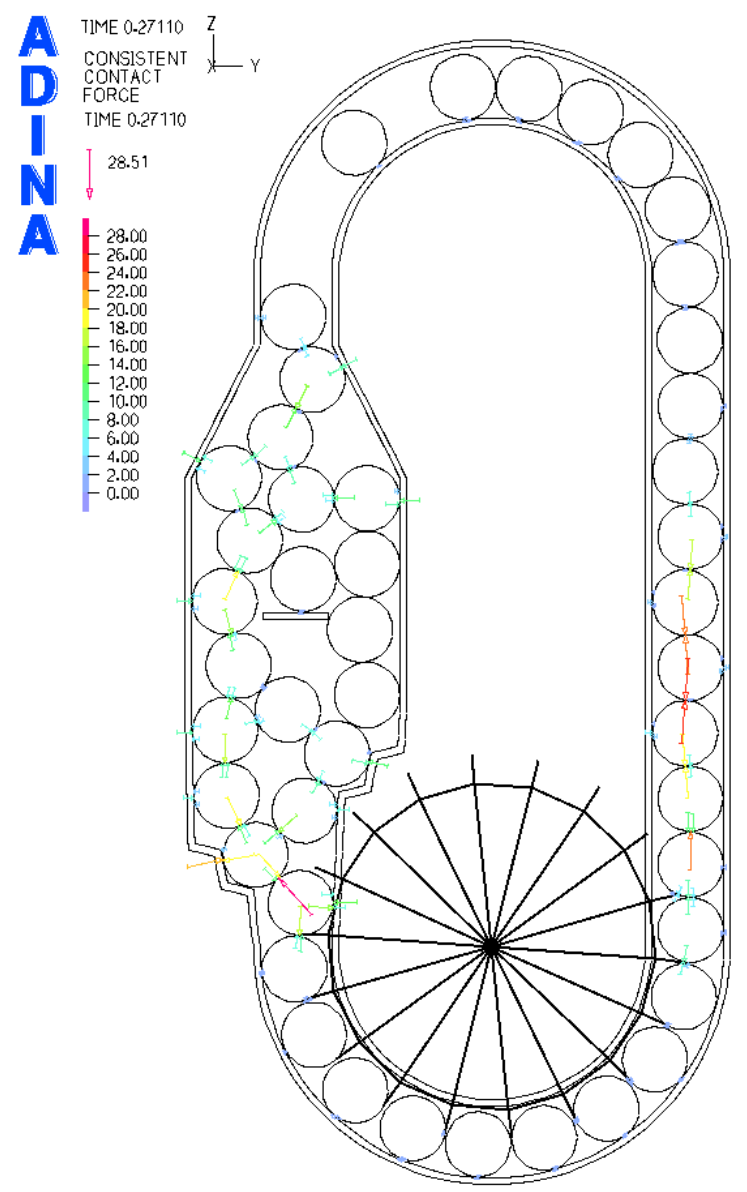

Fig. 4. Contact forces in time $0.2711 \mathrm{~s}[\mathrm{~N}]$ 
In numerical tests of the designed computational model, correctness of a design of a gradual constriction of the channel was proved. In this spot the mutual jamming of circular contact bodies does not take place.

Presented results of contact forces are shown in the time of 0.2711 in Figure 4. The speed of fluid flow is shown in Figure 5 in the same time; the speed of flow is shown in a form of velocity vectors $\mathrm{ms}^{-1}$.

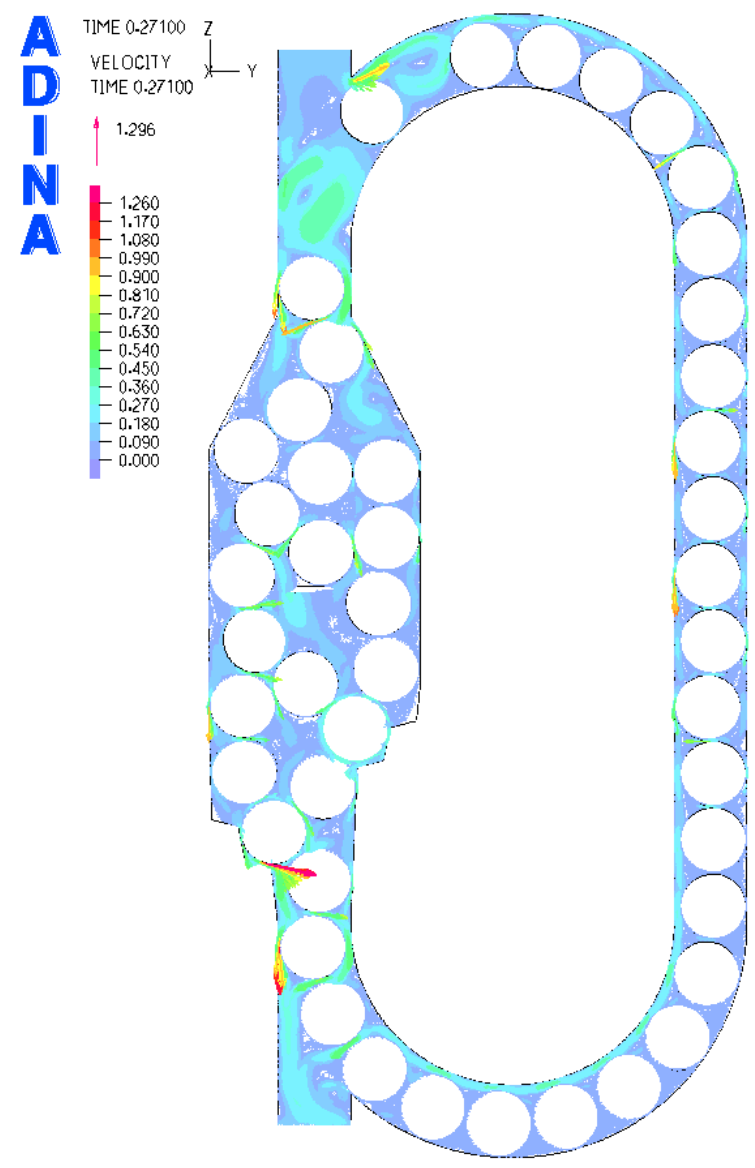

Fig. 5. Fluid flow velocity, vector results in time $0.2711 \mathrm{~s}\left[\mathrm{~ms}^{-1}\right]$

\section{Conclusion}

The designed FE computational model meets all required criteria imposed on the model. It enables a mutual interaction of contact bodies together with an interaction with channel boundaries. The interaction of bodies takes place in the flowing fluid, while the speed of fluid flow is possible to modify regardless the speed of rotation of auxiliary lines formed by beam elements.

In testing calculations was proved that a gradual channel shape is correctly designed and it enables gradual ordering of bodies into a taper part of the channel. In a transition of about 50 bodies their mutual jamming did not occur. 
The designed computational model enables a solution without a time limit, after completing of a computing task a restart of solution is possible and in the next solution of further time increment of a total solution.

This work is supported by: VEGA 1/0795/16 Development of effective methods for correction and optimization of coupled mechanical system.

This publication is the result of the project implementation: Development of optimal technology for analysis of limiting states of construction elements in contact ITMS: 26220220118 supported by the Operational Programme Research and development funded by the ERDF.

\section{References}

1. on-line manual, Adina, Theory and modelling guide, Volume I: Adina. (2016)

2. on-line manual, Adina, Theory and modelling guide, Volume III, CFD and FSI. (2017)

3. B. Ftorek, P. Tomasovic, B. Dorociakova, Comparison of two methods for solving nonlinear parabolic model in porous media. Proceedings of Aplimat 2009: 8th international conference, 259-263 (2009)

4. G. Domek, Research on the contact area between the timing belt and the toothed pulley. Lectures notes in engineering and computer science, 2242-2244 (2011)

5. J. Gerlici, T. Lack, Contact geometry influence on the rail/whel surface stress distribution, Procedia engineering 2, 2249-2257 (2010)

6. J. Vavro Jr., J. Vavro, P. Kovacikova, R. Bezdedova, Kinematic and dynamic analysis of the manipulator for removal of rough tyres. Procedia Engineering 136, 120-124 (2016)

7. M. Sofer, R. Halama, F. Fojtik, L. Vaclavek, Rolling contact fatigue research on VSBTUO, comparison of current test machine with it's new concept. New methods of damage and failure analysis of structural parts, 153-158 (2010)

8. J. Zapomnel, P. Ferfecky, A computational investigation of the disk-housing impacts of accelerating rotors supported by hydrodynamic bearings. Journal of applied mechanics-transactions of the ASME 78, (2011)

9. L. Jakubovičová, M. Sága, Computational analysis of contact stress distribution in the case of mutual stewing of roller bearing rings. Novel trends in production devices and systems 474, 363-368 (2014)

10. M. Astorino, J.F. Gerbeau, O. Pantz, K. F. Traoré, Fluid-structure interaction and multi-body contact: Application to aortic valves. Computer Methods in Applied Mechanics and Engineering 198, 3603-3612 (2009)

11. W. Schiehlen, Research trends in multibody system dynamics. Multibody System Dynamics 18, 3-13 (2007)

12. M. Nad', Modification of model properties of machining tool bodies. Proceedings of the $7^{\text {th }}$ international conference of DAAAM baltic industrial engineering, 1, 54-59 (2010)

13. I. Malujda, D. Wilczynski, Mechanical properties investigation of natural polymers. Procedia engineering 136, 263-268 (2016)

14. J. Zapoměl, V. Dekýš, P. Ferfecki, A. Sapietová, M. Sága, M. Žmindák, Identification of material damping of a carbon composite bar and study of its effect on attenuation of its transient lateral vibrations. Int. Journal of Applied Mechanics 7 (6), (2015) 